Nathaly Sarasty Narváez

Projeto Baseado em Confiabilidade de Vigas em Concreto Armado com e sem Reforço de Compósitos de Fibras de Carbono

Dissertação apresentada como requisito parcial para obtenção do título de Mestre pelo Programa de PósGraduação em Engenharia Civil da PUC-Rio.

Orientador: Profa. Marta de Souza Lima Velasco Co-orientador: Prof. Luiz Eloy Vaz 
Nathaly Sarasty Narváez

\section{Projeto Baseado em Confiabilidade de Vigas em Concreto Armado com e sem Reforço de Compósitos de Fibras de}

Carbono

Dissertação apresentada como requisito parcial para obtenção do título de Mestre pelo Programa de PósGraduação em Engenharia Civil da PUC-Rio. Aprovada pela Comissão Examinadora abaixo assinada.

Profa. Marta de Souza Lima Velasco

Orientador

Departamento de Engenharia Civil - PUC-Rio

Prof. Luiz Eloy Vaz

Co-Orientador

Universidade Federal Fluminense

Prof. Emil de Souza Sánchez Filho

Universidade Federal Fluminense

Prof. Júlio Jerônimo Holtz Silva Filho Departamento de Engenharia Civil - PUC-Rio

Profa. Andréia Abreu Diniz de Almeida Universidade Federal Fluminense

Prof. José Eugenio Leal

Coordenador Setorial do Centro Técnico Científico - PUC-Rio

Rio de Janeiro, 28 de fevereiro de 2012. 
Todos os direitos reservados. É proibida a reprodução total ou parcial do trabalho sem autorização da universidade, da autora e dos orientadores.

\section{Nathaly Sarasty Narváez}

Graduou-se em Engenharia Civil na Universidade de Nariño, Colômbia, em 2009. Na PUC-Rio desenvolveu seu trabalho de pesquisa com ênfase em concreto reforçado e análise de confiabilidade.

Ficha Catalográfica

Narváez, Sarasty Nathaly

Projeto baseado em confiabilidade de vigas em concreto armado com e sem reforço de compósitos de fibras de carbono / Nathaly Sarasty Narváez ; orientadores: Marta de Souza Lima Velasco, Luiz Eloy Vaz. - 2012.

146 f. il. (color.) ; $30 \mathrm{~cm}$

Dissertação (mestrado)-Pontifícia Universidade Católica do Rio de Janeiro, Departamento de Engenharia Civil, 2012.

Inclui bibliografia

1. Engenharia civil - Teses. 2. Análise de confiabilidade. 3. Vigas de concreto armado. 4. Compósitos de fibra de carbono. I. Velasco, Marta de Souza Lima. II. Vaz, Luiz Eloy. III. Pontifícia Universidade Católica do Rio de Janeiro. Departamento de Engenharia Civil. IV. Título. 
Este trabalho é dedicado a minha família por seu constante apoio e amor incondicional 


\section{Agradecimentos}

A Deus, pela oportunidade de desenvolver este trabalho no Brasil com sua benção e proteção diária.

Aos meus pais e irmã, exemplos de coragem e responsabilidade, pela dedicação, amor e acompanhamento em todos os meus empreendimentos. Seus conselhos me ajudaram a superar os momentos difíceis.

A minha avó Carmelita, tia Cristina e Vanessa, por me oferecerem sempre carinho e compreensão.

A minha orientadora professora Marta de Souza Lima Velasco por me dar a oportunidade de trabalhar com ela e me oferecer seus conhecimentos e sua confiança.

Ao meu co-orientador professor Luiz Eloy Vaz por compartilhar seus conhecimentos de engenharia, por me receber na sua casa e me mostrar a belíssima cultura brasileira através da música e da historia.

Aos professores do departamento de Engenharia Civil da PUC-Rio e aos técnicos do Laboratório de Estruturas e Materiais.

A os amigos que conheci durante o mestrado com os quais formamos uma segunda família por tantas alegrias e boas experiências, especialmente a Vanessa e Eliot por estarem comigo em todo momento transmitindo valores como o respeito, a tolerância e a paciência.

Ao CNPq e a PUC-Rio pelo seu apoio financeiro. 


\section{Resumo}

Narváez, Sarasty Nathaly; Velasco, Marta de Souza Lima. Eloy, Luiz Vaz. Projeto Baseado em Confiabilidade de Vigas em Concreto Armado com e sem Reforço de Compósitos de Fibras de Carbono. Rio de Janeiro, 2012. 146p. Dissertação de Mestrado - Departamento de Engenharia Civil, Pontifícia Universidade Católica do Rio de Janeiro.

No Brasil, o dimensionamento de estruturas de concreto armado segue as recomendações da norma brasileira NBR 6118-2003. A norma brasileira, usa a metodologia de dimensionamento semi-probabilístico que não quantifica a probabilidade de falha de um elemento estrutural. A análise de confiabilidade de estruturas é uma ferramenta que possibilita o cálculo da probabilidade de falha associada a um estado limite e também um dimensionamento para uma determinada probabilidade de falha denominada probabilidade de falha alvo. Este trabalho tem como objetivo desenvolver uma metodologia que possibilite o dimensionamento baseado em confiabilidade de seções de vigas de concreto armado sem e com reforço com compósitos de fibras de carbono. Na análise de confiabilidade desenvolvida foram consideradas como variáveis aleatórias o carregamento, as resistências do aço e do concreto, e a resistência à tração dos compósitos de fibras de carbono com propriedades estatísticas determinadas com base nos resultados dos ensaios realizados no laboratório de Estruturas e Materiais do Departamento de Engenharia Civil da PUC-Rio (LEM-DEC). Exemplos de dimensionamento de seções de vigas de concreto armado submetidas à força cortante e à flexão foram realizados inicialmente por métodos semi-probabilísticos e depois utilizando a metodologia proposta baseada em confiabilidade. A análise dos resultados mostra a viabilidade de um projeto baseado em confiabilidade usando um índice de probabilidade de falha determinado para cada tipo de projeto aliando economia e segurança.

\section{Palavras-chave}

Análise de Confiabilidade; Vigas de Concreto Armado; Compósitos de Fibra de Carbono. 


\section{Abstract}

Narváez, Sarasty Nathaly; Velasco, Marta de Souza Lima (Advisor); Eloy, Luiz Vaz (Co-advisor). Reliability-based Design of RC Beams Strengthened with CFC. Rio de Janeiro, 2012. 146p. Dissertation - Departamento de Engenharia Civil, Pontifícia Universidade Católica do Rio de Janeiro.

In Brazil, the design of reinforced concrete structures follows the recommendations of Brazilian standard NBR 6118-2003. The Brazilian standard uses the methodology of semi-probabilistic design that does not quantify the probability of failure of a structural element. The reliability analysis of structures is a tool that allows the calculation of probability of failure associated with a limit state and also the design for a given probability of failure. This work aims to develop a methodology that enables the Reliability-based-design of concrete beams sections with and without strengthening with carbon fiber composites. In the reliability analysis were considered as random variables the loads, the compression concrete strenght, yield strenght, and tensile strength of carbon fiber composites with certain statistical properties based on the results of tests performed in the Structures and Materials laboratory at PUC-Rio (LEM-DEC). Examples of reinforced concrete beams strengthened with CFC were performed initially by semi-probabilistic methods and then using the proposed methodology based on reliability. The analysis shows the feasibility of a Reliability-baseddesign using a reliability index of failure probability determined for each type of project combining economy and safety.

\section{Keywords}

Reliability Analysis; Reinforced Concrete; Carbon Fiber Composites. 


\section{Sumário}

1 Introdução $\quad 22$

1.1. Considerações Gerais 23

1.2. Revisão Bibliográfica 25

1.2.1. Estudos sobre Vigas de Concreto Armado Reforçadas com CFC Solicitadas à Força Cortante 25

1.2.2. Estudos sobre Vigas de Concreto Armado Reforçadas com CFC Solicitadas à Flexão 25

1.2.3. Estudos de Análise de Confiabilidade em Vigas de Concreto Armado Reforçadas com CFC $\quad 32$

1.3. Objetivos 34

1.4. Organização do Trabalho 35

2 Projeto de Vigas de Concreto Armado Submetidas à Força Cortante $\begin{array}{ll}\text { e à Flexão } & 37\end{array}$

2.1. Projeto de Vigas de Concreto Armado Submetidas à Força Cortante 37

2.1.1. Critério do Projeto da NBR 6118-2003 40

2.2. Projeto de Vigas de Concreto Armado Submetidas à Flexão 43

2.2.1. Expressões de Dimensionamento de Vigas Submetidas à Flexão 46

2.3. Projeto de Vigas de Concreto Armado Reforçadas com CFC Submetidas à Força Cortante 48

2.3.1. Modelo de Chen e Teng (2003 a, b) 50

2.3.2. Modos de Ruptura à Força Cortante 57

2.4. Projeto de Vigas de Concreto Armado Reforçadas com CFC Submetidas à Flexão $\quad 59$

2.4.1. Coeficiente de Segurança do CFC para Flexão 60

2.4.2. Modelo de Cálculo do Reforço à Flexão com CFC 61

2.4.3. Modos de Ruptura à Flexão de Vigas Reforçadas 63

3 Confiabilidade de Estruturas $\quad 66$

3.1. Níveis dos Métodos de Projeto de Estruturas 66 
3.2. Conceitos Fundamentais $\quad 69$

3.2.1. Função Densidade e Cumulativa de Probabilidade 69

3.2.2. Correlação entre Variáveis Aleatórias 70

3.2.3. Função de Falha 72

3.2.4. Probabilidade de Falha 73

3.2.5. Confiabilidade de Estruturas 73

3.3. Definição do Índice de Confiabilidade Beta 74

3.3.1. Índice de Confiabilidade de Referência $\quad 75$

3.4. Métodos de Análise de Confiabilidade 77

3.4.1. Método de Simulação de Monte Carlo 77

3.4.2. Métodos Analíticos de Análise de Probabilidade $\quad 81$

3.4.2.1. Método FORM (First Order Reliability Method) 82

3.5. Confiabilidade de Sistemas Estruturais $\quad 85$

3.5.1. Sistemas em Paralelo $\quad 86$

3.5.2. Sistemas em Série $\quad 86$

3.5.3. Índice de Confiabilidade do Sistema

3.5.4. Limites para a Probabilidade de Falha do Sistema 89

3.6. FERUM, (Finite Element Reliability Using Matlab) 90

3.7. Medidas de Sensibilidade $\quad 91$

4 Determinação Experimental das Propriedades Estatísticas das Fibras de Carbono 93

4.1. Objetivos dos Ensaios $\quad 93$

4.2. Sistema de Reforço Estrutural 94

4.3. Descrição do Ensaio de Resistência à Tração das Fibras de Carbono 96

4.4. Determinação do Tipo de Distribuição e Parâmetros Estatísticos das $\begin{array}{ll}\text { Variáveis } & 101\end{array}$

4.4.1. Teste de Kolmogorov-Smirnov (K-S) 101

$\begin{array}{ll}\text { 4.4.2. Teste do Chi-quadrado } & 104\end{array}$

$\begin{array}{ll}\text { 4.5. Resultados } & 108\end{array}$

5 Formulação do Problema 109

5.1. Introdução 109

5.2. Funções de Falha para Análise de Confiabilidade de Vigas Submetidas à 
Força Cortante

5.2.1. Funções de Falha para Análise de Confiabilidade de Vigas Submetidas à Força Cortante sem Reforço

5.2.2. Funções de Falha para Análise de Confiabilidade de Vigas Submetidas à Força Cortante com Reforço

5.3. Funções de Falha para Análise de Confiabilidade de Vigas Submetidas à Flexão

5.3.1. Função de Falha para Análise de Confiabilidade de Vigas Submetidas à Flexão sem Reforço

5.3.2. Função de Falha para Análise de Confiabilidade de Vigas Submetidas à Flexão com Reforço

5.4. Exemplos de Aplicação

5.4.1. Exemplo de Viga Submetida à Força Cortante e à Flexão Analisada por Seções

5.4.2. Exemplo de Viga com e sem Reforço de CFC

5.4.2.1. Propriedades Estatísticas das Variáveis

5.4.2.2. Exemplo de Viga Submetida à Força Cortante

5.4.2.3. Exemplo de Viga Submetida à Flexão

6 Conclusões e Sugestões para Trabalhos Futuros 


\section{Lista de Figuras}

Figura 1.1 - Compósitos com fibras de carbono (CFC); retirada de Juvandes (2002).

Figura 2.1 - Trajetória das tensões principais em viga não fissurada. 37

Figura 2.2 - Distribuição de tensões e fissuras de força cortante. 38

Figura 2.3 - Modelo treliça; adaptada de Collins e Mitchell (1987). 39

Figura 2.4 - Modelo de uma viga de concreto segundo a treliça generalizada. 40

Figura 2.5 - Diagrama de deformações dos domínios 2, 3 e 4 . 44

Figura 2.6 - Zonas de dimensionamento em função da deformação no aço. 44

Figura 2.7 - Distribuição de tensões e deformações em viga de seção

retangular com armadura simples. $\quad 46$

Figura 2.8 - Configurações de reforço à força cortante com CFC; retirada de Khalifa (1999).

Figura 2.9 - Viga reforçada; adaptada de Chen e Teng (2003a). 50

Figura 2.10 - Detalhe do reforço contínuo; adaptada de Chen e Teng (2003a) 51

Figura 2.11 - Força cortante x abertura da fissura; adaptada de

Teng et al.(2001) 54

Figura 2.12 -Testes realizados por Chen e Teng; adaptada de Chen e Teng

(2003 a). 55

Figura 2.13 - Posições eficazes ou ineficazes das faixas de PRF; adaptada de Chen e Teng (2003a).

Figura 2.14 - Modos de ruptura à força cortante; adaptada de Täljsten (2003) 58

Figura 2.15 - Modos de ruptura ao cortante da viga reforçada; adaptada de

Täljsten (2003).

Figura 2.16 - Diagramas $\sigma$ x $\varepsilon$ parábola-retângulo e retangular simplificado para distribuição de tensões de compressão no concreto. 61

Figura 2.17 - Modos de Ruptura possíveis; adaptada de Beber (2003). 64

Figura 3.1 - Funções: (a) densidade; (b) cumulativa de probabilidades. $\quad 70$

Figura 3.2 - Função densidade de probabilidades conjunta. 71 
Figura 3.3 - Definição da função de falha.

Figura 3.4 - Índice de confiabilidade $\beta$

Figura 3.5 - Algoritmo para o cálculo da probabilidade de falha por Monte Carlo.

Figura 3.6 - Transformação do espaço original para o espaço reduzido (Normal

Padrão); adaptada de Choi e Youn (2001).

Figura 3.7 - Aproximação do Método FORM para superfícies côncavas e convexas.

Figura 3.8 - Representação gráfica da busca do ponto de projeto para um problema com duas variáveis; adaptada de Choi e Youn (2001).

Figura 3.9 - Sistemas na análise de confiabilidade a) sistema em série

b) sistema em paralelo.

Figura 3.10 - Função de falha com mais de um ponto de mínimo.

Figura 4.1 - Tecido de fibras de carbono no sentido longitudinal

(Meneghel, 2005).

Figura 4.2 - Resina epóxi: (a) componentes A e B; (b) preparação da resina para a aplicação. (Meneghel, 2005).

Figura 4.3- Dimensões dos corpos-de-prova segundo a norma ASTM D3039/3039M.

Figura 4.4 - Confecção dos corpos-de-prova.

Figura 4.5 - Máquina de ensaios EMIC.

Figura 4.6 - Extensómetro colado no corpo-de-prova. 98

Figura 4.7 - Corpos-de-prova ensaiados. 99

Figura 4.8 - Modos de falha dos corpos-de-prova. $\quad 99$

Figura 4.9 - Teste Kolmogorov-Smirnov $F_{x}\left(x_{i}\right)$ vs $S n . \quad 102$

Figura 4.10 - Função densidade acumulada teórica e observada da resistência última à tração do CFC. 103

Figura 4.11 - Frequências teóricas e observadas teste Chi-quadrado. 105

Figura 4.12 - Histograma teste Chi-quadrado. 106

Figura 4.13 - Funções de distribuição Normal, Lognormal e Weibull. 106

Figura 5.1 - Dimensões da viga analisada. 112

Figura 5.2 - Variação da carga concentrada na viga. 113

Figura 5.3 - Índices de confiabilidade e variação da posição da carga concentrada na seção 1 . 
Figura 5.4 - Índices de confiabilidade e variação da posição da carga concentrada na seção 2.

Figura 5.5 - Índices de confiabilidade e variação da posição da carga concentrada na seção 4 .

Figura 5.6 - Índices de confiabilidade e variação da posição da carga concentrada na seção 4.

Figura 5.7 - Índices de confiabilidade e variação da posição da carga concentrada na seção 5 .

Figura 5.8-Exemplo proposto.

Figura 5.9 - Diagrama de força cortante para as condições iniciais.

Figura 5.10 - Diagrama de momentos de flexão para as condições iniciais. 12

Figura 5.11 - Diagrama de força cortante após mudança de uso. 121

Figura 5.12 - Diagrama de momento de flexão após mudança de uso. $\quad 121$

Figura 5.13 - Gráfico do processo iterativo do cálculo da armadura de cisalhamento e valores de $\beta_{1}, \beta_{2}, \beta_{\text {alvo }}$ e $\beta_{\text {sistema }}$.

Figura 5.14 - Representação gráfica do processo iterativo do cálculo do CFC e $\beta_{1}, \beta_{3}, \beta_{4}, \beta_{\mathrm{s}}$.

Figura 5.15 - Fatores de importância da viga submetida à força cortante sem reforço.

Figura 5.16 - Fatores de importância viga submetida à força cortante com reforço

Figura 5.17 - Gráfico do processo iterativo do cálculo da armadura de flexão e valores de $\beta_{1}$ e $\beta_{\text {alvo }}$.

Figura 5.18 - Fatores de importância viga submetida à flexão sem e com reforço 


\section{Lista de Tabelas}

Tabela 1.1 - Propriedades das fibras de carbono (Bulletin 14 da FIB, 2001). 24

Tabela 2.1 - Modos de ruptura possíveis (Beber, 2003).

Tabela 3.1 - Índices de confiabilidade $\beta$ alvos relativos a um ano de período de referência.

Tabela 4.1 - Recomendações para a geometria dos corpos-de-prova segundo ASTM D3039/3039M.

Tabela 4.2 - Resultados dos ensaios.

Tabela 4.3 - Resultados teste Kolmogorov-Smirnov.

Tabela 4.4 - Resultados teste Chi-Quadrado para a resistência à tração do CFC

Tabela 5.1- Variáveis aleatórias com propriedades estatísticas

Tabela 5.2 - Índices de confiabilidade para a seção 1.

Tabela 5.3 - Índices de confiabilidade por FERUM e Monte Carlo.

Tabela 5.4 - Índices de confiabilidade para a seção 2.

Tabela 5.5 - Índices de confiabilidade para a seção 3.

Tabela 5.6 - Índices de confiabilidade para a seção 4.

Tabela 5.7 - Índices de confiabilidade para a seção 5.

Tabela 5.8 - Desvio padrão segundo condição de preparo NBR-6118.

Tabela 5.9 - Cargas acidentais em lajes.

Tabela 5.10 - Variáveis do exemplo.

Tabela 5.11 - Variáveis aleatórias do exemplo com os parâmetros estatísticos. 127

Tabela 5.12 - Comparação métodos Monte Carlo e FORM.

Tabela 5.13 - Iteração de cálculo da armadura a cisalhamento $\beta_{1} e \beta_{2}$.

Tabela 5.14 - Variáveis aleatórias do exemplo com os parâmetros estatísticos. 131

Tabela 5.15 - Valores dos índices de confiabilidade $\beta_{1}, \beta_{3}, \beta_{4}$ para a taxa de $\operatorname{CFC} \rho_{\mathrm{f}}$.

Tabela 5.16 - Iteração de cálculo da taxa de fibra de carbono e $\beta_{\text {sistema }}$.

Tabela 5.17 - Fatores de importância viga submetida à força cortante sem reforço. 
Tabela 5.18 - Fatores de importância viga submetida à força cortante com reforço

Tabela 5.19 - Variáveis aleatórias do exemplo com os parâmetros estatísticos 135

Tabela 5.20 - Iteração de cálculo da armadura a flexão $\beta_{1}$.

Tabela 5.21 - Fatores de importância viga submetida à flexão sem e com reforço 


\section{Lista de Símbolos}

\section{Romanos}

$A_{f} \quad$ Área de CFC

$A_{s} \quad$ Área de armadura longitudinal de aço

$A_{s w} \quad$ Área de armadura transversal de aço

$b_{w} \quad$ Largura da seção transversal da viga

CoV Coeficiente de variação

d Altura útil da viga

$D_{f} \quad$ Fator de distribuição da tensão

$D_{n} \quad$ Diferença máxima entre as funções de densidade acumulada teórica e observada

$D_{n}{ }^{\alpha} \quad$ Diferença máxima entre as funções de densidade acumulada teórica e observada aceitável

$d_{f} \quad$ Altura efetiva do CFC

$d_{f b} \quad$ Distância entre a face comprimida da viga e a extremidade inferior do CFC

$d_{f t} \quad$ Distância entre a face comprimida da viga e a extremidade superior do CFC

$E_{f} \quad$ Módulo de elasticidade do CFC

$E_{s} \quad$ Módulo de elasticidade do aço

$f_{c d} \quad$ Resistência de cálculo do concreto a compressão

$f_{c j} \quad$ Resistência média do concreto à compressão, prevista para a idade de $\mathrm{j}$ dias

$f_{c k} \quad$ Resistência característica do concreto à compressão

$f_{c m} \quad$ Resistência média do concreto à compressão (cilindro padrão)

$f_{c t d} \quad$ Resistência de cálculo do concreto à tração direta

$f_{c t k, \text { inf }}$ Resistência característica inferior do concreto à tração

$f_{c t m} \quad$ Resistência média do concreto à tração

$f_{f} \quad$ Tensão última do CFC

$f_{f e} \quad$ Tensão efetiva do CFC

$f_{f} \quad$ Valor médio da resistência à tração última do CFC

$f_{c}^{\prime} \quad$ Resistência característica do concreto à compressão 
$f_{R}(r) \quad$ Função densidade de probabilidade marginal da resistência

$f_{R S}(r, s)$ Função densidade de probabilidades conjunta

$f_{S}(s) \quad$ Função densidade de probabilidade marginal da solicitação

$f_{y} \quad$ Tensão de escoamento à tração do aço

$f_{y d} \quad$ Tensão máxima permitida no aço

$f_{y k} \quad$ Resistência característica de escoamento do aço

$f_{y w} \quad$ Resistência de escoamento da armadura transversal de aço

$f_{y w d} \quad$ Resistência de cálculo de escoamento da armadura transversal de aço

$f_{x}(x) \quad$ função densidade de probabilidades de $X$

$F_{x}(x)$ função cumulativa de probabilidades de $X$

$g_{1} \quad$ Função de falha relativa à ruína das diagonais comprimidas de concreto

$g_{2} \quad$ Função de falha relativa à ruína por tração diagonal

$g_{3} \quad$ Função de falha relativa à ruína por tração diagonal, devido à ruptura do reforço.

$g_{4} \quad$ Função de falha relativa à ruína por tração diagonal, devido ao descolamento do reforço.

$h \quad$ Altura da viga

$h_{f e} \quad$ Altura efetiva do CFC

$L_{e} \quad$ Comprimento de ancoragem efetivo

$L_{m a x} \quad$ Comprimento de aderência máximo

$M_{d} \quad$ Momento fletor de cálculo

$M_{d m a x} \quad$ Momento fletor de cálculo máximo no trecho em análise

$M_{p p} \quad$ Momento na viga submetida à flexão devida ao peso próprio

$P_{i} \quad$ Termo de primeira ordem da probabilidade de falha de um sistema em série

$P_{i k} \quad$ Termo de segunda ordem da probabilidade de falha de um sistema em série

$P_{i k l} \quad$ Termo de terceira ordem da probabilidade de falha de um sistema em série

$p_{f} \quad$ Probabilidade de falha

$p_{\text {fadm }} \quad$ Probabilidade de falha admissível

$R_{c c} \quad$ Resultante de tensão do concreto na flexão 
$R_{s t} \quad$ Resultante de tensão do aço na flexão

$s \quad$ Espaçamento entre as armaduras transversais de aço

$S_{d} \quad$ Desvio padrão da dosagem do concreto, tabelado na NBR 1265

(1996) de acordo com a condição de preparo do concreto

$s_{f} \quad$ Espaçamento de eixo a eixo entre estribos de CFC

$s_{\text {fmax }}$ Espaçamento de eixo a eixo entre estribos de CFC

$s_{\text {nom }} \quad$ Valor característico da resistência de escoamento do aço

$t_{f} \quad$ Espessura da faixa de CFC

$\operatorname{Var}(X) \quad$ Variância de uma variável aleatória

$V_{c} \quad$ Parcela da força cortante resistida pelo concreto

$V_{c d} \quad$ Valor de projeto da parcela da força cortante resistida pelo concreto

$V_{f} \quad$ Parcela da força cortante resistida pelo CFC

$V_{f d} \quad$ Valor de projeto da parcela da força cortante resistida pelo CFC

$V_{f, d} \quad$ Parcela de força cortante resistida pelo CFC, limitada pelo descolamento do compósito

$V_{f, r} \quad$ Parcela de força cortante resistida pelo CFC, limitada pela ruptura do compósito

$V_{g} \quad$ Força cortante proveniente do carregamento permanente

$V_{R d l} \quad$ Valor de projeto da parcela da força cortante resistida pelo concreto

$V_{R d 2}$ Valor de projeto da força cortante resistida pelas bielas comprimidas

$V_{R d 3} \quad$ Força cortante resistente de cálculo

$V_{S} \quad$ Força cortante solicitante

$V_{S d} \quad$ Força cortante solicitante de cálculo

$V_{s w} \quad$ Parcela da força cortante resistida pela armadura transversal de aço

$V_{s w d} \quad$ Valor de projeto da força cortante resistida pela armadura transversal de aço

$w_{f} \quad$ Largura do estribo de CFC

$z_{c c} \quad$ Braço de alavanca

$z_{b} \quad$ Coordenada da extremidade inferior

$z_{t} \quad$ Coordenada da extremidade superior

$X_{2 l i m} \quad$ Limite do domínio de deformação na flexão 


\section{Gregos}

$\alpha \quad$ Ângulo de inclinação entre a armadura transversal de aço e o eixo longitudinal da viga

$\alpha_{i} \quad$ Cosseno diretor entre o vetor normal à superfície de falha no ponto de projeto $\mathrm{y}^{*}$ e o eixo da variável reduzida $\mathrm{Y}_{\mathrm{i}}$

$\alpha_{i}, \alpha_{k} \quad$ Vetores dos cossenos diretores nos pontos de projeto $\mathrm{y}^{*}$ associados aos modos de ruptura

$\lambda \quad$ Comprimento máximo de ancoragem

$\beta \quad$ Ângulo de inclinação entre a orientação das fibras do CFC e o eixo longitudinal da viga

$\beta \quad$ Índice de confiabilidade

$\beta_{\text {alvo }} \quad$ Índice de confiabilidade admissível

$\beta_{1} \quad$ Índice de confiabilidade referente ao esmagamento das bielas de compressão

$\beta_{2} \quad$ Índice de confiabilidade referente à ruína por tração diagonal

$\beta_{3} \quad$ Índice de confiabilidade referente à tração diagonal devido à ruptura do reforço

$\beta_{4} \quad$ Índice de confiabilidade referente à tração diagonal devido ao descolamento do reforço

$\beta_{\text {serie }}$ Índice de confiabilidade equivalente obtido considerando a formulação se sistemas em série

$\beta_{L} \quad$ Coeficiente que traduz o comprimento de ancoragem efetivo

$\beta_{w} \quad$ Coeficiente que reflete o efeito da razão entre a largura do CFC e do concreto

$\xi \quad$ Relação entre as coordenadas das extremidades inferior e superior do reforço.

$\varepsilon_{c d} \quad$ Deformação de encurtamento na fibra mais comprimida de concreto

$\varepsilon_{c u} \quad$ Deformação de encurtamento última na fibra mais comprimida de concreto

$\varepsilon_{f} \quad$ Deformação específica do CFC

$\varepsilon_{f e} \quad$ Deformação específica efetiva do CFC

$\varepsilon_{f u} \quad$ Deformação específica última do CFC

$\varepsilon_{\text {máx }}$ Deformação específica máxima, ou limite, do CFC 


\begin{tabular}{|c|c|}
\hline$\varepsilon_{s}$ & Deformação do aço do gráfico esforço - deformação \\
\hline$\varepsilon_{s d}$ & Deformação de alongamento na armadura tracionada \\
\hline$\varepsilon_{y d}$ & Deformação de alongamento na armadura tracionada \\
\hline$\phi$ & Fator de minoração da resistência \\
\hline$\Phi()$ & Função de distribuição cumulativa da variável normal padrão \\
\hline$\Phi(,, \rho$ & Função de distribuição cumulativa bidimensional normal padrão \\
\hline$\gamma_{c}$ & Fator de segurança do concreto \\
\hline$\gamma_{f}$ & Coeficiente de segurança para resistência de ancoragem \\
\hline$\gamma_{m}$ & Coeficiente de minoração do momento \\
\hline$\gamma_{s}$ & Fator de segurança do aço \\
\hline$\theta$ & Inclinação das bielas de compressão \\
\hline$\rho$ & Matriz de correlação \\
\hline$\rho_{f}$ & Taxa geométrica de CFC \\
\hline$\rho_{i k}$ & $\begin{array}{l}\text { Coeficiente de correlação entre dois modos de ruptura (duas } \\
\text { funções de estado) }\end{array}$ \\
\hline$\rho_{s w}$ & Taxa geométrica de armadura transversal \\
\hline$\rho_{x, y}$ & Coeficiente de correlação \\
\hline$\sigma_{f m a ́ x}$ & Tensão máxima no CFC \\
\hline$\sigma_{f M a x \_} D$ & Tensão máxima no FRP, limitada pelo descolamento do compósito \\
\hline$\sigma_{f M a x \_R}$ & Tensão máxima no FRP, limitada pela ruptura do compósito \\
\hline$\sigma_{f y w}$ & Desvio padrão da resistência de escoamento do aço \\
\hline$\sigma_{s}$ & Esforço a tração do aço do gráfico esforço - deformação \\
\hline$\sigma_{s d}$ & Tensão de cálculo na armadura tracionada \\
\hline
\end{tabular}




\section{Lista de Abreviaturas}

\begin{tabular}{|c|c|}
\hline PUC-Rio & Pontifícia Universidade Católica do Rio de Janeiro \\
\hline CFC & $\begin{array}{l}\text { Carbon fibers composites (Compósitos com Fibras } \\
\text { de carbono) }\end{array}$ \\
\hline $\mathrm{ACI}$ & American Concrete Institute \\
\hline FIB & Federation Internationale du Beton \\
\hline NBR & Norma Brasileira \\
\hline AFORM & Advanced First-Order Reliability Method \\
\hline FORM & $\begin{array}{l}\text { First Order Reliability Method (Método de } \\
\text { Confiabilidade de primeira ordem) }\end{array}$ \\
\hline PRF & Polímero reforçado com fibras \\
\hline CFRP & $\begin{array}{l}\text { Carbon Fibre Reinforced Polymer (Polímero } \\
\text { Armado com Fibras de Carbono) }\end{array}$ \\
\hline JCSS & Joint Committee on Structural Safety \\
\hline LRFD & Load and Resistance Factor Design \\
\hline PDF & $\begin{array}{l}\text { Probability Density Function (Função Densidade de } \\
\text { Probabilidades) }\end{array}$ \\
\hline $\mathrm{CDF}$ & $\begin{array}{l}\text { Cumulative Distribution Function (Função de } \\
\text { Distribuição Cumulativa) }\end{array}$ \\
\hline FOSM & $\begin{array}{l}\text { First Order Second Moment (Método de } \\
\text { Confiabilidade de segundo momento) }\end{array}$ \\
\hline FERUM & Finite Element Reliability Using Matlab \\
\hline $\mathrm{UCB}$ & Universidade da Califórnia em Berkeley \\
\hline RBDO & Reliability-Based Design Optimization \\
\hline LEM-DEC & Laboratorio de Estruturas e Materias do \\
\hline
\end{tabular}

